\title{
Qualitative research: extending the range with flexible pattern matching
}

\section{Ricarda B. Bouncken ${ }^{1}$ D $\cdot$ Yixin Qiu ${ }^{1}$ D $\cdot$ Noemi Sinkovics $^{2}$ D $\cdot$ Wolfgang Kürsten $^{3}$}

Received: 16 November 2020 / Accepted: 29 January 2021 / Published online: 16 February 2021

(c) The Author(s) 2021

\begin{abstract}
The flexible pattern matching approach has witnessed increasing popularity. By combining deduction with induction in logic, flexible pattern matching is well suited for exploration and theory development. The paper discusses its logic, advantages and process of this approach while offering a review of research adopting this approach. We also compare and contrast it with another popular qualitative data analysis technique, the grounded theory approach, to further ground the method on the established knowledge and elaborate its strength and fitting context. This paper advances the flexible pattern matching approach by suggesting a five-step roadmap to conduct qualitative research with the approach.
\end{abstract}

Keywords Flexible pattern matching - Qualitative methods · Grounded theory • Connecting theories and data $\cdot$ Qualitative rigor

JEL Classification B40 · C53

Yixin Qiu

Yixin.Qiu@uni-bayreuth.de

Ricarda B. Bouncken

Bouncken@uni-bayreuth.de

Noemi Sinkovics

noemi.sinkovics@auckland.ac.nz

Wolfgang Kürsten

wolfgang.kuersten@uni-jena.de

1 Chair for Strategic Management and Organization, University of Bayreuth, Prieserstraße 2, 95444 Bayreuth, Germany

2 Management and International Business, University of Auckland, 12 Grafton Rd, Auckland Central 1010, New Zealand

3 Chair for Finance, Banking and Risk Management, Friedrich Schiller University of Jena, Carl-Zeiß-Straße 3, 07743 Jena, Germany 


\section{Introduction}

Qualitative techniques are widely applied in business and management research (Cassell and Bishop 2019; Gioia et al. 2013; Strauss and Corbin 1998). The principal reason is that qualitative studies can provide rich insights that explain underlying mechanisms and processes (Grund and Walter 2015; Muhic and Bengtsson 2019). Table 1 lists some of the recent qualitative studies published in Review of Managerial Science. However, there has been criticism with respect to their replicability and rigor. To address such criticism, a range of criteria and techniques were developed over the past decades (Cassell et al. 2018; Langley and Abdallah 2011). Whereas some researchers welcome these more structured guiding principles (Eisenhardt 1989; Gioia et al. 2013; Yin 1994b), others voice concerns about a potential loss of creativity (Langley and Abdallah 2011).

The pattern matching framework put forward by Sinkovics (2018) creates a balance between the extremes of rigid standardization and complete anarchy. The framework organizes the diverse qualitative techniques into different categories based on the degree of the pattern matching. Pattern matching is both a logic and a technique. As a logic, the aim is to identify and describe patterns with as much accuracy as possible. However, while some patterns can be described and interpreted with a high level of accuracy, others can only be interpreted with a certain level of probability (Hammond 1966). The reason comes from the observers' interpretation; they do it through a path-dependent lens developed through their past experiences and based on the amount of available information at the time of the observation. As a technique, pattern matching applies this logic and provides a set of guidelines with respect to how to make the researchers' internal mental models as explicit as possible. In this way, the approach provides readers with a roadmap to reproduce the researchers' thought processes and how they arrived at the conclusions they present in their manuscript.

This pattern matching framework allows the organization of the diverse qualitative techniques into three overarching categories; namely partial pattern matching, full pattern matching, and flexible pattern matching. Partial pattern matching aims to engage the investigator's mental models in the theorizing process. Grounded theory, as put forward by Glaser and Strauss (1968), and the Gioia (2004) method of structural coding represent examples of bottom-up partial pattern matching. The purpose is to start with the data and identify patterns that emerge from it. Top-down partial pattern matching employs visualization techniques to specific bodies of literature to identify innovative research questions (Sinkovics 2016). Full pattern matching generally includes all statistical methods (Trochim 1985; Trochim and Donnelly 2008). In the qualitative domain, full pattern matching entails the operationalization of multiple theoretical explanations for a specific phenomenon to determine which explanation is the most accurate (Yin 2009; Yin and Moore 1988).

In contrast, flexible pattern matching spans the space between partial and full pattern matching. It allows the interaction of deductive and inductive components, thus combining rigor with a high level of flexibility. Eisenhardt's (1989) 


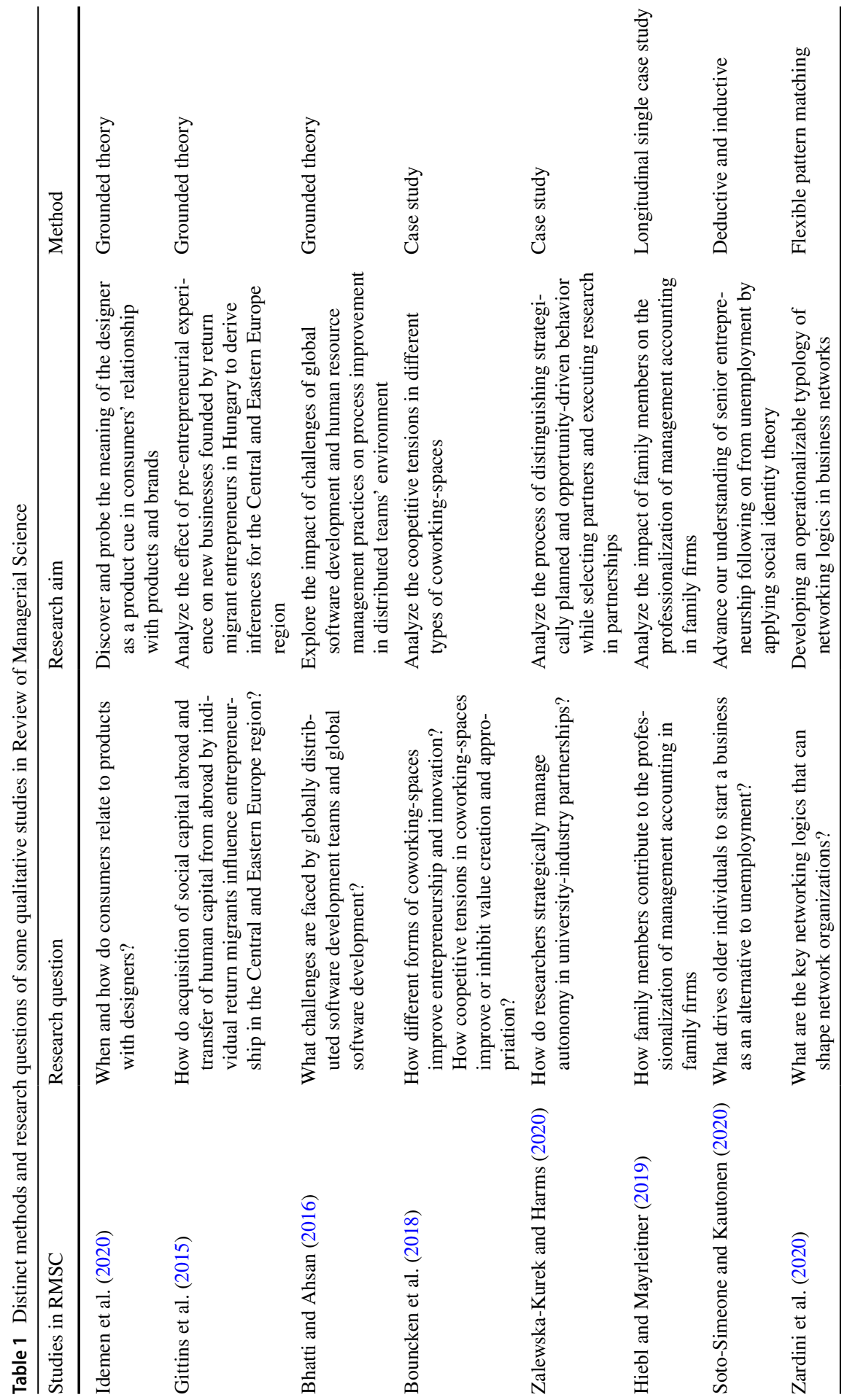




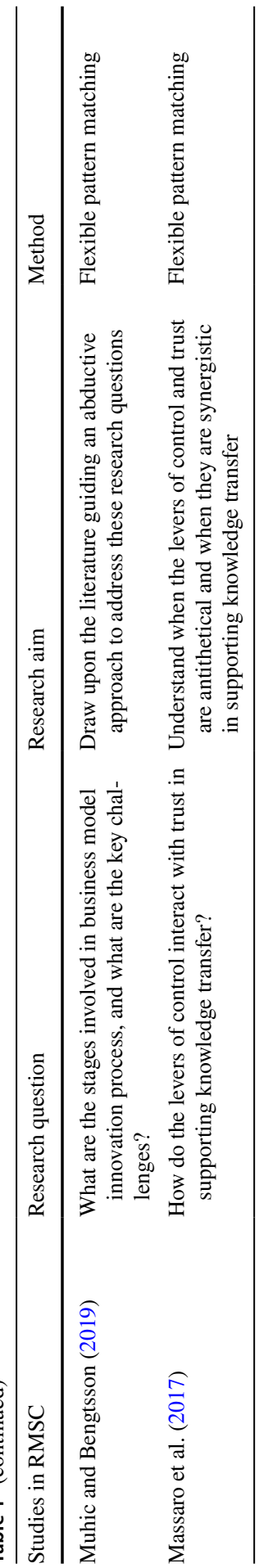


theory building approach from cases and King's (2004) template analysis are examples of the flexible pattern matching logic. Recently, there is an emerging body of work in this category that builds on a systematic or semi-systematic review of the literature to define the initial theoretical patterns that are then matched to the data (Bouncken and Barwinski 2020; Sinkovics et al. 2014, 2019). Further theorizing and/or theory development is then triggered by mismatches between theoretical patterns and observed patterns, or by the emergence of unexpected observed patterns (Bouncken and Barwinski 2020; Bouncken et al. 2021). However, before Sinkovics (2018) systematically categorized pattern matching into the three overarching categories, scholars did not differentiate between these distinct approaches, referring to all three as pattern matching. In this paper, to ensure the consistent use of terms and avoid confusion, we refer to the approach that involves the flexible pattern matching logic as "flexible pattern matching".

Despite or rather because of the growing number of studies applying the principles of this type of flexible pattern matching (Bouncken and Barwinski 2020; Gatignon and Capron 2020; Sinkovics et al. 2014, 2019), there is a need to gain more insights into its foundation, its relation to established qualitative methods, and how it best informs theory building. Moreover, the growing questions on the welldeveloped grounded theory also call for avenues with more rigorous analysis processes and stronger relationships with the existing literature (Cuervo-Cazurra et al. 2016).

This paper aims to provide a comprehensive understanding and guideline for conducting qualitative research with a flexible-pattern-matching design. We first present and explain the general logic of the flexible pattern matching approach and its advantages, augmented by explaining prior studies adopting flexible pattern matching. Then, we analyze its combination and differences with an established but also questioned qualitative method, grounded theory, to further elaborate on the flexible pattern matching approach and its strength, as well as to extend our discussion on the approach to a broader context of qualitative studies. Subsequently, we develop a roadmap for conducting qualitative studies with the method. Finally, future research directions are discussed and how the flexible pattern matching approach can help to gather more insights towards future theory development.

\section{Explaining the flexible pattern matching approach}

Flexible pattern matching involves the iterative matching between theoretical patterns derived from the literature and observed patterns emerging from empirical data (Sinkovics 2018). Its particular focus on the interplay between theoretical and empirical patterns distinguishes it from the "Gioia Method", which focuses on capturing informant meaning, and the "Eisenhardt Method", which focuses on sharpening particular settings distinction (Langley and Abdallah 2011). The aim of flexiblepattern-matching studies is to explore how inconsistencies and breakdowns derived from the pattern-matching process can help to problematize and develop theoretical ideas (Alvesson and Kärreman 2007; Shane 2000) or how consistencies can help to test or expand the contextual boundaries of existing theories (Ross and Staw 1993). 
Theoretical patterns and observed empirical patterns are two key components in flexible pattern matching. A pattern indicates the arrangement of objects or entities that are non-random and describable (Trochim 1989). Following this logic, all theories are built on patterns, but they are also different from patterns. Completed theories contain structural relationships that enable the generation of predictions as predicted patterns. For example, based on prior work about behavioral difficulties of mergers and hostility of employees, Greenwood et al. (1994) developed two hypotheses on how organizational (in)compatibility will escalate from the courtship stage to the consummation stage in the merging process.

Similarly, observed patterns are distinct from collected data; they are "constellations" of observations or social logics whose independence and ordering compose items that can be identified as a pattern (Jancsary et al. 2017). For example, in Shane's (2000) study on how entrepreneurs discover opportunities, three patterns are generated from extensive and multiple-source data on eight firms, explaining the effect from the dissemination of information, situation-specific superiority, and prior knowledge.

However, theoretical and observed patterns are developed from distinct processes. Theoretical patterns are deduced from related theories, while observed empirical patterns emerge through continuous iteration and comparison between theoretical patterns and collected data (Bitektine 2008; Bouncken et al. 2021; Sinkovics et al. 2019). This matching technique infuses the analysis and theorizing process with both imagination and discipline. That is, empirical materials create room for the emergence of new, unexpected dimensions, as well as revision of the theoretical patterns. Simultaneously, relevant theories create relative boundaries to anchor the theorizing process in specific perspectives and areas, prohibiting spurious ideas (Alvesson and Kärreman 2007; Sinkovics et al. 2019). The process of flexible pattern matching, therefore, consists of the deduction of a set of theoretical patterns from prior studies, the formation of actual observed patterns through the lens of these theoretical patterns, and the comparison between them that enables the emergence of new patterns. The resulting theoretical model or framework may represent a revision and/or extension of the initial theoretical patterns (Gatignon and Capron 2020; Greenwood et al. 1994; Sinkovics et al. 2014, 2019). A more detailed roadmap for this process will be discussed later.

The adoption of flexible pattern matching can be found in studies on organizational culture (Ainsworth and Cox 2003; Canato et al. 2013; Montealegre 2002), entrepreneurship (Chiles et al. 2007; Shane 2000), institutional logic (Jancsary et al. 2017; Reay and Jones 2016; Van de Ven and Huber 1990), and organizational pathways (Bouncken and Barwinski 2020; Gatignon and Capron 2020; Rindova et al. 2007). The approach has five key advantages that advocate its more frequent application, especially in research areas where there are suitable theories and prior studies to deduce initial theoretical patterns.

First, the idea of relating several pieces of information from the collected data to some theoretical proposition can strengthen the internal validity of case studies (Eisenhardt 1989; Yin 1994a). To compare a predicted theoretical pattern with an observed empirical pattern, scholars need to design a rich theoretical framework based on existing studies guiding the research design. The initial framework and the 
comparison legitimate the establishment of a causal relationship and guide the generation of observed patterns from collected data (Massaro et al. 2017). This advantage represents the foundation for the other four advantages. For example, in Massaro et al. (2017) study, they developed a matrix with dependent and independent variables that served as a collection of theoretical patterns. The later comparison between the matrix and the collected data increased the reliability of the findings on the joint effect of trust and control mechanisms on knowledge transfer in the networks of small and medium-sized enterprises.

Second, the interplay between theories and observations in flexible pattern matching allows readers to follow the researcher's thought processes from the conceptualization stage to the data interpretation stage. A sound conceptual background at the front end of the manuscript provides a roadmap for the data analysis as well as the articulation of the findings. This is because it aids the researcher to go beyond a mere description of divergent empirical findings (Brooks and King 2014; Brooks et al. 2015; Thornton et al. 2012). Therefore, readers are better able to make theoretical sense of 'complex phenomena' while comprehending and evaluating the findings in relation to prior work (Sinkovics et al. 2014, 2019). For instance, Gatignon and Capron (2020) adapted the eight-dimension principles of polycentric governance from Ostrom (2005) as their analytical framework to examine how firms address voids in emerging markets. The eight principles unraveled the differences and relationship between the two theoretical patterns and the observed pattern, and therefore offers a structural process for readers to apprehend the articulated theoretical implication and comprehend findings in relation to prior work.

Third, the matches and mismatches between theoretical and observed patterns, as well as the emergence of unexpected patterns, provides the researcher with a structure for theorizing about the findings. While a mismatch between the two sets of patterns may initially appear to be a "defect", in fact, it represents an "opportunity" to revise or extend existing theory or initiate the theorizing process towards a new theory. For example, in Bouncken and Barwinski's (2020) study on knowledge ties in global digital business, they first detected potential modification in the theoretical patterns through collected data, then they conducted a second-round of pattern matching to analyze the cases with mismatches and the causes behind. This twostage flexible pattern matching facilitates the identification of strategies to grow a global business in the 3D printing industry and leads to the introduction of the concept of shared digital identity.

Fourth, the approach is effective in capturing categories for analysis. The systematic or semi-systematic review of the relevant literature helps researchers engage in a priori theorizing process. In other words, researchers can engage in thought experiments based on the existing literature in terms of what they expect the observed patterns to be. This a priori framework also provides a "grammar" for data analysis that describes the direction of analysis while allowing for emergent themes from cases and data. For instance, to reveal the complex constitution of organizational culture and dynamics of control, Ainsworth and Cox (2003) developed initial patterns including three interpretive divisions from the literature and the concept of shared understandings of interpretative divisions from Parker (1995) to further develop and refine the typology. The comparison of the initial patterns with the collected data 
showed that the meaning and implications of these divisions varied depending on the local context, vis-à-vis the complex dynamics of control. Ainsworth and Cox (2003: p. 1469) described the initial patterns as "a generalizable 'grammar"" that is "flexible enough to explore how similar dynamics could yield local and particular meanings across the cases".

Five, the flexible pattern matching approach has substantial promise in longitudinal and/or single-case studies. The a priori patterns as theoretical interpretation provide material for replication and comparison, and thus represent a powerful way to gain insights from a single case. For example, Ross and Staw's (1993) goal was to ground an escalation theory further to address the theory void at an organizational level by testing two propositions (as theoretical patterns) derived from the literature, and also to explore the organizational escalation and exit process. Therefore, they conducted a longitudinal single-case study with a pattern-matching approach for both theory testing and development. By examining the archival and interview data of the case, Ross and Staw not only discerned fit with the a priori propositions, supporting the tested theories, but also developed an understanding of organizational escalation, exit, and how it is resolved. Analyzing the single case enabled Ross and Staw (1993) to "comprehend as fully as we could the events of Shoreham and to match those events with potential models of escalation and exit" through "moving back and forth between the empirical data and possible theoretical conceptualization".

\section{Flexible pattern matching and grounded theory: comparison and bridging}

Grounded theory is one of the most cited qualitative research methods in business and organization studies (Gioia et al. 2013; Glaser and Strauss 1968; Langley 1999; Strauss and Corbin 1998). It is a technique for surfacing and constructing relevant categories that provide deep and rich theoretical descriptions of the context to explain the phenomenon of interest and clarify all data-to-category connections (Chiles et al. 2010; Gioia et al. 2013; Soto-Simeone and Kautonen 2020). Flexible pattern matching similarly involves the process of "coding" data where researchers distill insights from qualitative data into more manageable "categories" or "patterns". Although flexible pattern matching starts with an a priori development of codes/patterns/constructs from the literature, it also allows the a posteriori development of codes that are completely data-driven (Sinkovics 2018). In contrast, grounded theory starts with an inductive exploration of the data (Langley 1999; Sang and Sitko 2015), the literature is consulted during and after the coding process (Kenealy 2012). Although partly intertwined, flexible pattern matching and grounded theory differ in multiple aspects. The grounded theory has reached wide legitimacy from its development and adoption while also received increasing critiques in the recent year. In this section, we discuss the connections and differences between the flexible pattern matching and the grounded theory (as summarized in Table 2), to further introduce the approach 


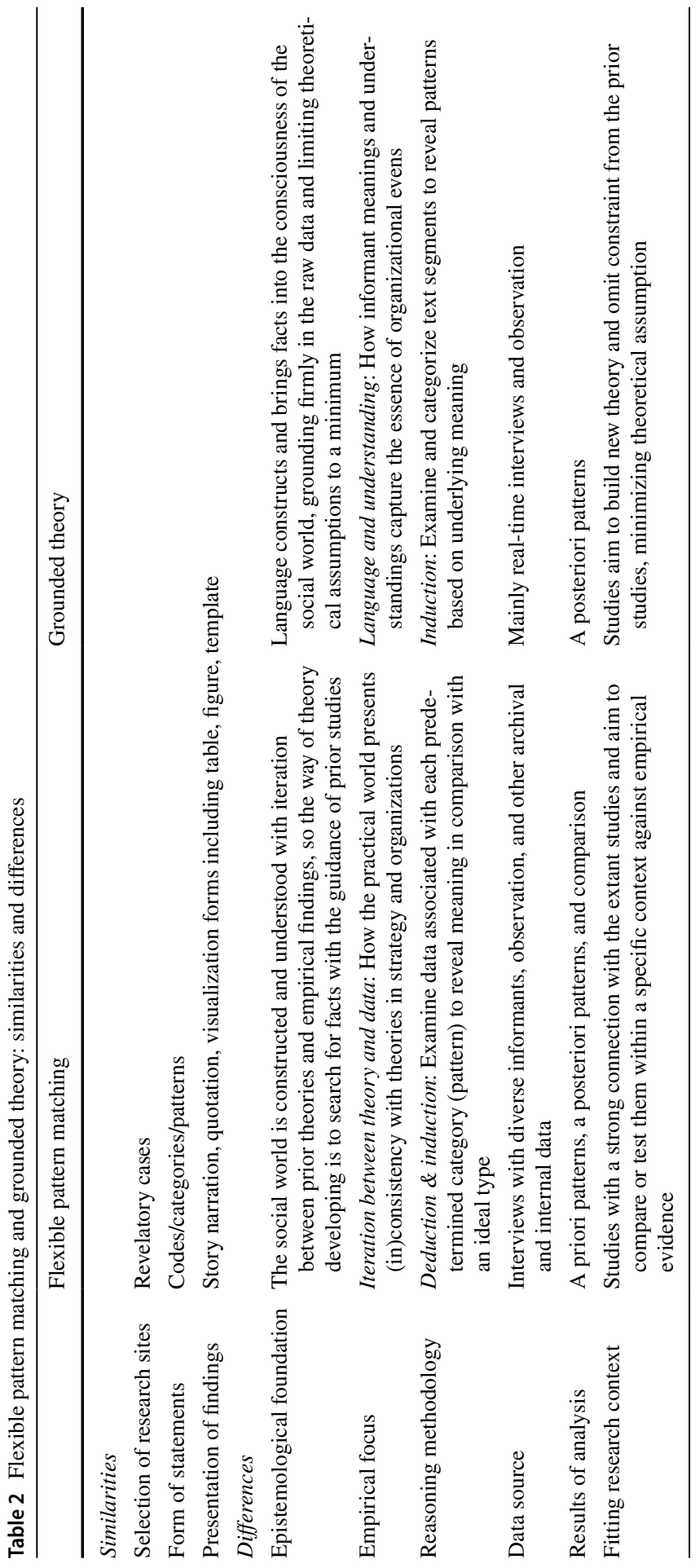


by drawing on the established method and address the features of the approach to the recent call for more rigorous qualitative methods (Cuervo-Cazurra et al. 2016; Langley and Abdallah 2011).

The shared features of the flexible pattern matching and the grounded theory approaches mostly originate in the process of exploring the meaning of certain social phenomena or activities that are entangled with the context. Studies adopting either flexible pattern matching or the grounded theory approach commonly investigate revelatory research sites, instead of critical or extreme ones (Langley and Abdallah 2011; Yin 1994a), where they believe that they may find interesting answers to the research question with rich and trustworthy data. After selecting relevant case(s), researchers gather empirical data-ranging from interviews, archive data to ethnographic observations - to gain an understanding of settings and events from insiders', and maybe also other stakeholders', experience, opinions, and explanations. In data analysis, both methods involve organizing nuanced descriptions or statements into categories or patterns that show the particular logic. In the final presentation of these findings, both methods favor providing a visualized representation with templates, figures, or tables. A widely accepted example of structuring and visualizing data connected to grounded theory is the Gioia method. It introduces a highly disciplined coding and analyzing process, presenting output with a threeorder hierarchical data structure in a tree-shaped fashion (Gioia et al. 2010, 2013; Langley and Abdallah 2011).

Although there are similar components in these two methods, they have different epistemological foundations. Flexible pattern matching builds on the assumption that the way we understand the social world is constructed through iterations between prior theories and empirical observations, so the way of theory development is to compare and contrast prior knowledge with empirical observations (Burchell and Kolb 2003; Reay and Jones 2016; Sinkovics 2018). In contrast, grounded theory is driven by an interpretive philosophy. It is based on the assumption that "reality is a constructed and shifting entity and social processes can be created and changed by interactions among people" (Grbich 2013: 80). Symbols, signs, and language are used to achieve meaning. Prior theoretical assumptions are limited to a minimum to leave room for the construction of meaning from raw data (Gioia et al. 2013; McCutcheon and Meredith 1993; Strauss and Corbin 1998). Grounded theory is best suited under three conditions: (1) When there is very little or no prior knowledge of the phenomenon and thus a new theory is needed to explain it, (2) when the focus of the investigation is on a microcosm of interaction in a specific setting where all related aspects need to be examined, or (3) when the purpose of the research is to build new theoretical explanations to explain changes in a field (Grbich 2013).

However, outside of these three conditions, as Eisenhardt states, a "clean theoretical slate" approach is not convincing enough, since the whole research process, including site selection, data collection, and analysis, is guided by specific rationale and research questions, indicating at least some theoretical basis and voids to address (Eisenhardt 1989; McCutcheon and Meredith 1993). Further, if grounded theory is used in settings other than the ones described above, it presents challenges to draw generalizations and a "substantive" theory beyond the specific research context (Brooks et al. 2015; Langley 1999). Therefore, outside 
of the three categories where the use of grounded theory is needed, flexible pattern matching is better suited as a method.

A particular feature of the flexible pattern matching approach is its use of a priori patterns, building on existing theories, developing ideas from relevant studies, and ensuring focus on the investigated area (Baxter and Berente 2010; Burton and Khammash 2010; Cuervo-Cazurra et al. 2016). This feature is an essential difference between flexible pattern matching and ground theory derived from their distinct epistemological foundation. This difference is also represented in Sinkovics' (2018) pattern-matching framework, where grounded theory is classified as a partial pattern matching technique.

The use of a priori patterns or constructs in flexible pattern matching can be especially advantageous in qualitative studies as they provide a clear rationale and logic for data collection and analysis (Brooks et al. 2015; Waring and Wainwright 2008). On the other hand, the a priori patterns are themselves subject to revision, redefinition, changing scopes, or other types of modification, so that leaves space for exploration and emergence of new themes (Burchell and Kolb 2003; McCutcheon and Meredith 1993; Sinkovics 2018). Multiple iterations between theories and empirical evidence is another feature of flexible pattern matching linked to the previous one. The data analysis, namely the matching of theoretical and observed patterns, requires particular attention to the interplay between theories and data, by focusing on how insights from empirical data break down or challenge those theoretical constructs (Alvesson and Kärreman 2007; Chiles et al. 2010). This strong connection between prior studies and collected data also enhances the generalizability of the generated findings and developed theory since the theoretical base functions as a counterpart for comparison and as external verification. Further, flexible pattern matching also allows the emergence of empirical patterns from the data. This inductive element adds to the already flexible method and allows the extension of theories by complementing them with new concepts. Therefore, flexible pattern matching can be adapted to a broad range of studies (Corsaro and Snehota 2010; Waring and Wainwright 2008).

It is important to note, however, that grounded theory and flexible pattern matching are not mutually exclusive. The inductive element within the flexible pattern matching approach makes it suitable to create a reverse flexible pattern matching design. This represents a bridge between grounded theory and flexible pattern matching. A good example of this is Chiles et al.'s (2010) study on organizational emergence. The authors started with grounded theory, aiming to develop new theoretical insights on the emergence of organizational collectives. However, after realizing the substantial fit between their data-driven framework and the literature on complexity theory, they shifted to a flexible pattern matching approach, aiming to compare the empirical framework with complexity theory. The switch to complexity theory enables the study to complement complexity theory with a collective-level perspective by matching and adding to the patterns from complexity theory with the field data (Chiles et al. 2010). 


\section{Research with flexible pattern matching: stages and roadmap}

While the flexible pattern matching approach enjoys increasing popularity in qualitative business and management studies, there is a need to provide further guidelines for performing it. To this end, we provide a synthesis of steps identified by going through prior studies employing this technique. We suggest five critical steps outlined in Fig. 1. We also present five ways to present pattern-matching results.

\subsection{Formulating the research question(s)}

An initial definition of the research question(s) is a critical start of studies with a flexible-pattern-matching design, and also of general explorative studies (Cassell and Bishop 2019; Cassell et al. 2006; Sinkovics 2018). If the researcher is struggling to identify a meaningful research question, a top-down partial pattern matching technique is recommended, as outlined by Sinkovics (2016). The researcher can then build on the results of this pre-literature review literature analysis to theoretically ground their research question for their flexible pattern matching study. Theorydriven research questions are generally derived from identified voids or conflicts in the extant literature. The contribution of the study then rests on the rich and in-depth qualitative data that offers insights to answer the questions (Eisenhardt 1989; Eisenhardt and Graebner 2007). For example, Sinkovics et al. (2014) first constructed a research gap in the organizational literature on a deep understanding of how the base-of-the-pyramid business models and social value creation are related. They then applied a flexible-pattern-matching logic to conduct field research by linking

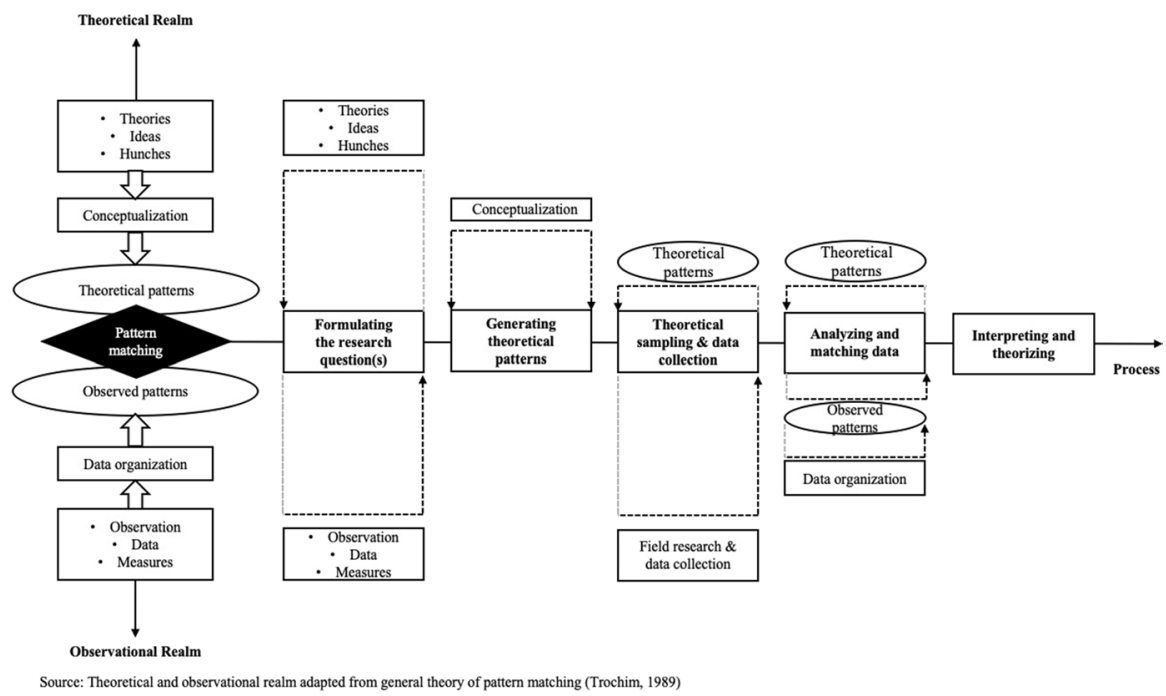

Fig. 1 Iteration between theoretical and observational realm in conducting flexible-pattern-matching research 
it to extant studies. Phenomenon-driven research questions highlight the importance of a specific phenomenon and deficiency of plausible extant theory to explain it, contributing by complementing or challenging existing theories (Eisenhardt 1989; Eisenhardt and Graebner 2007). For example, Gatignon and Capron (2020) motivated their study with the observation that a fast-expanding company in Brazil overcame institutional challenges in a way distinct from two patterns suggested by the literature. Whether theory-driven or phenomenon-driven, this initial grounding is of great importance in flexible pattern matching to convince readers of the study's contribution to business and theory building or testing. This is in addition to demonstrating a lack of or insufficient evidence from the current literature to address it. Moreover, it is equally significant to know that the research questions are tentative and can be modified along with more insights derived from the study (Sinkovics 2018).

\subsection{Generating theoretical patterns}

In flexible pattern matching, patterns/constructs/templates deduced from the theories are constructed and documented before the data collection (Bitektine 2007; Kauppila 2010; Sinkovics et al. 2019). The generation of a priori patterns from preexisting theoretical knowledge or the construction of a conceptual framework differs from widely-adopted qualitative study methods, including the grounded theory and the Gioia method. This feature enables studies to have a firm grounding in related literature, shape the initial theoretical design of the research, and design the interview guide that seamlessly feeds into the data analysis. Researchers should also consider the scope and extension of the initial patterns because too many predefined patterns might hamper the analysis and incite exploration out of bounds, while at the other extreme, too few patterns may neglect insights from relevant studies (King 2004; King, Brooks, \& Tabari, 2018). We suggest following a well-defined research focus and conducting an exhaustive review of relevant studies to form an appropriate number of patterns that fit the study's objective, as in Sinkovics et al. (2019) and Bouncken and Barwinski (2020). To develop an initial concept model, Bouncken and Barwinski (2020) built on knowledge exchange, digitalization, and global business literature and identified five forms of global knowledge exchange, four mechanisms of exchange, and two possible outcomes. The initial conceptual model generates an overall picture of the extant patterns on the investigated topic.

\subsection{Theoretical sampling and data collection}

The previous procedure provides the theoretical basis for the following field research and establishes a connection between theories and practical social activities. Therefore, case or object selection is determined by the theoretical base and the nature and accessibility of social activities. With the generated theoretical patterns as a foundation, researchers adopt theoretical sampling to select revelatory cases that are particularly suitable for examining, testing, or extending the logics indicated by the established patterns. The sample of flexible pattern matching studies can be a 
single case or multiple cases. The choice of a single case is mostly based on the uniqueness, revelation of the given case, and its potential in developing theories. In contrast, multiple cases are selected for theoretical relevance, aiming to achieve replication, diverse profiles, alternative explanations, or extension of theory (Eisenhardt 1989; Eisenhardt and Graebner 2007; Yin 1994a). For example, Ross and Staw (1993) chose the Shoreham case to achieve both theory testing and development because of its fit with the research focus on organizational escalation and exit, as well as the uniqueness of this case in terms of scale, amount of investment, and the escalated result. In contrast, Saka (2004) selected three cases one large and two medium-sized Japanese companies in the UK to study the process of cross-national diffusion of work systems. The aim was to conduct a rigorous comparison of the diffusion process through flexible pattern matching. In estimating the correct time, sites, and informants for data collection, researchers should follow the guidance of theoretical focus and the information from industrial experts. Multiple data sources can be collected and combined to meet the need of specific research aims. In-depth interviews, observation, documents, and artifacts are four primary sources of qualitative data; Table 3 provides an overview of key types and corresponding functions.

\subsection{Analyzing and matching data}

The appearance of data analysis and matching after theoretical sampling and data collection does not necessarily mean that it should be conducted after the completion of the previous step. When possible, a constant comparison approach is recommended. This entails a process of simultaneous data collection and data analysis (Eisenhardt and Graebner 2007) to enable researchers to glean predominant and characteristic patterns from data and take advantage of flexible data collection (Muhic and Bengtsson 2019). The analysis process in flexible pattern matching is iterative and involves moving back and forth between theoretical patterns and the empirical data to discern the observed patterns. Inconsistencies with observed patterns or the emergence of unexpected patterns may trigger a further literature analysis that in turn can drive further explorations of the data (Montealegre 2002; Muhic and Bengtsson 2019; Shane 2000). For example, Sinkovics, Hoque, and Sinkovics (2016) operationalized two theoretical concepts, social upgrading and social value creation, as the initial theoretical template to identify the overlaps and discrepancies with the observed patterns. The matching and comparison results enabled the authors to demonstrate that social upgrading can destroy pre-existing social value. In the flexible pattern matching approach, the analysis saturation is reached when the matching process reveals adequate information to answer the research question that has been observed, and new insights stop appearing (Glaser and Strauss 1968; Montealegre 2002; Strauss and Corbin 1998). It is also possible that at a certain stage of the data analysis, the data collection method undergoes alteration, addition, or modification. This change might be needed, especially in explaining future developments as well as in research aiming at theory-building, because the iteration between theory and empirical observations might induce a new line of thinking, or new data collection opportunities might arise. However, this flexibility should be channeled 


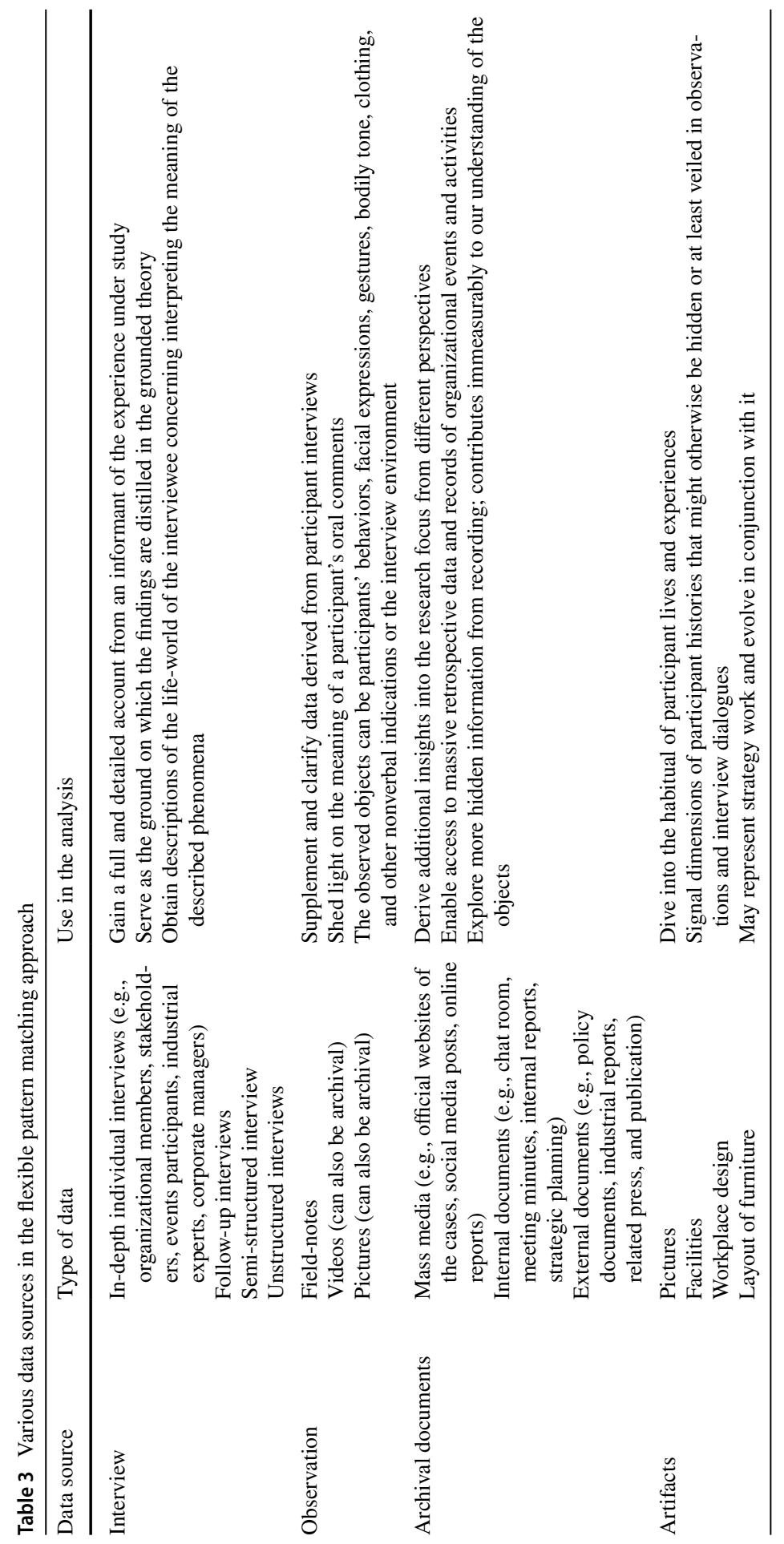


into controlled alterations aiming at better grounding the theory or providing additional, relevant, and valuable theoretical insights. Arbitrary, researchers should avoid opportunistic modifications.

\subsection{Interpreting and theorizing}

In studies adopting a flexible pattern matching approach, findings and space for theory development derive from the mismatch between theoretical pattern and observed empirical patterns (Bouncken and Barwinski 2020; Sinkovics et al. 2019), the match between modified theoretical framework and empirical observation (Jobber and Lucas 2000), or the emergence of new, unexpected patterns from the data (Sinkovics et al. 2014). In line with general qualitative studies, the presenting of findings derived from a flexible pattern matching approach also involves story narration interspersed with quotations from informants and other supporting materials. However, the interpretation of insights from this approach is neither constrained in relating the narrative of each case, resulting in theory losing and text balloons, nor trapped in generating spurious insights. Rather, the articulation of findings from flexible pattern matching is structured by theoretical patterns or a theoretical framework that enables the comparison between theoretical and empirical patterns. This comparison involves asking what is consistent, what is inconsistent, in which way, and why. Multiple approaches to present the comparison and findings from flexible pattern matching have been adopted by researchers, including template analysis, tables, matrix, figures, and narratives. In the following paragraphs, we will briefly introduce each of them along with studies adopting each form of presentation.

Template analysis is a category of well-developed flexible pattern matching logic (Crabtree and Miller 1999; King 2004; King et al. 2018). Template analysis deploys hierarchical coding to "produce a list of codes ('template') representing themes identified in their text data" (King 2004). The initial themes and sub-themes derived from the literature review and were revised in the light of the ongoing analysis of empirical data when mismatches between theoretical and empirical patterns, in the form of templates, appears. For example, in their study on service delivery work in complex case management, Spurrell et al. (2019) began with developing a template from the literature, including three themes that explain the network context: patient network perspective, commissioner network perspective, and clinical team network perspective. Their empirical data analysis suggests adding network interconnection as an additional perspective for understanding and researching context functioning.

Making use of tables is an efficient way to compare sets of patterns and present insights from the findings. In most cases, a framework derived from prior studies is used as the measure or underlying analytical structure for comparison. The advantage of the form of tables is that it systematically presents the contrast of patterns from attribute to attribute. For instance, in the light of Ostrom's eight design principles, Gatignon and Capron (2020) evaluated two patterns from the extant literature and one pattern from the case regarding how they address each of the principles and the degree of their consistency with the principles. It distinctly presents how the new 
pattern from the case is different from the others and how it contributes to addressing voids in market-based institutions.

Matrix is a variation of tables, with numbers or other forms of measurement scales to operationalize the distance or closeness between the initial patterns and the observed ones. For example, Sinkovics et al. (2019) provide an overview of corresponding operationalization to theoretical patterns, expected observed patterns, and empirically observed patterns with the evaluation of low, medium or high, aiming to "identify potential 'breakdowns' in our understanding based on current frameworks and/or empirical findings".

Using figures enables the presentation and visualization of more complex relationships and interactions between constructs. For example, to examine the integrating view of technical, political, and cultural aspects on firms' marketing performance, Jobber and Lucas (2000) first developed a modified version of Tichy's (1983) framework based on a review of the literature. Subsequently, they mapped out the relationships among the elements by using a graphical representation. After that, they created another figure with the same structure to indicate the causes and linkages from the empirical cases. They used the comparison between these two figures to support their theorizing.

Narration is the most fundamental and informative way of presenting the findings from flexible pattern matching that can complement and interlink with all the aforementioned approaches. There are a number of prior studies that provide instructive insights on presenting verbal narration as the "art of words" (cf. Bansal and Corley 2012; Graebner et al. 2012; Pratt 2009).

There is no one-size-fits-all approach to craft high-quality write-ups of flexiblepattern-matching studies. Nevertheless, by relying on the general logic of flexible pattern matching (Sinkovics 2018), researchers can tailor this roadmap to fit the requirements of their particular study.

\section{Challenges}

Flexible pattern matching is a qualitative research approach based on the linkage and iteration between theory and observation. Although this design allows improved rigor in qualitative studies through a strong grounding in extant theories without sacrificing the rich insights and exploration from qualitative data, these advantages still come at some cost related to challenges for the researcher(s). First, the researcher might encounter difficulties in deducing convincing, inclusive, and focused theoretical patterns from various theoretical perspectives. It initially requires a carefullyconsidered research aim, followed by a specified but comprehensive literature search within this sphere (Brooks and King 2014). To further aid controlled deduction (Jancsary et al. 2017; Trochim 1989), researchers can draw on the expertise of coauthors as indicated by the roadmap in this study. A main issue is to consider that words and sentences as the medium of logical deduction should not weaken the logic of the reasoning process of theoretical pattern deduction (Lee 1989). As Lee (1989) stated, "deductions with verbal propositions (i.e., qualitative analysis) therefore only 
deprive itself of the convenience of the rules of algebra; it does not deprive itself of the rules of formal logic".

Second, applying flexible pattern matching involves an exceptionally large commitment of time and effort to generate sets of patterns and repeatedly move back and forth between them (Reay and Jones 2016). As the matching process is based on the development of both theoretical and observed patterns, it necessitates an exhaustive literature review on certain research topics, insight generation therefrom, and iteration between pieces of empirical data and theoretical patterns. When longitudinal investigation and data are included, then the research is further associated with a substantial time span and tracing on the formation of the focal events that matter for the study. A team-based approach to conducting such arduous work can help ensure the research project's continuity and eliminate bias.

Third, some researchers might be concerned about the generalizability of the insights from studies adopting a flexible pattern matching approach. This is, in fact, a common discussion on some other qualitative methods. One can address this by articulating the object of interest within a context that includes a range of related elements for adopting the theory (Langley 1999; Trochim 1989). As Allison and Zelikow (1971) argued in their book, "refining partial paradigms, and specifying the classes of actions for which they are relevant, maybe a more fruitful path to limited theory and propositions than the route of instant generalization". Further, while adopting the flexile pattern matching approach, the theoretical patterns derived from the literature and multiple perspectives can increase the external vitality of the findings and insights. Ultimately, the aim of flexible pattern matching is to pave the way for meaningful large-scale studies.

\section{Suggestions and future developments}

Business administration, as an application-oriented discipline inside the humanities and social sciences, nowadays has recognized the need for methodological openness. Qualitative research and methodological pluralism have proved to expand the discipline's practical relevance in recent times considerably. As flexible pattern matching rests on an iterative linkage between theoretical patterns, heterogeneous qualitative techniques, and empirical observation, this approach may serve as a promising qualitative research design to improve rigor especially in those fields of business administration that, unlike humanities-related fields like, say, organizational theory or general management, show a strong resemblance to the natural sciences. For example, equilibrium models of asset pricing or firm financing in perfect capital markets typically rely on mathematics (e.g., arbitrage theory Harrison and Kreps 1979; Ross 1978)) or physics (e.g., stochastic processes like Geometric Brownian motion, (Black and Scholes 1973)). In practice, however, the state of equilibrium will often not be attained due to limits of arbitrage (Shleifer and Vishny 1997). In fact, empirical evidence sheds doubt on the basic assumption that investors behave rationally but are misguided by noisy external signals, path-dependent loss of confidence during the arbitrage process, and cognitive dissonances induced by framing effects and mental accounting (Barberis and Thaler 2002; De Long et al. 
1990). These inconsistencies between theoretical and observed patterns result in a bidirectional relationship between single facts and empirical observations on the one side and contextual framing effects on the other side.

The flexible pattern matching approach, although becoming increasingly popular, is still in a developing stage. Therefore, this paper contributes by synthesizing insights from existing studies with respect to its logic, application, advantages, as well as a comparison and combination with grounded theory-based approaches. We are also providing a set of guidelines on the process of theory development. Researchers from multiple domains have recognized the promise of this approach for theorizing and theory building. Specifically, the continuous iteration between theoretical and observational realms (as presented in Fig. 1) leverages and balances both the in-depth investigation from qualitative data and the underlying rationale derived from the literature (Zardini et al. 2020). Thus, this approach paves the way to identify, problematize, revise, and complement existing theories. The advancement in theories lays the foundation for more convincing and valid strategies in business and management studies. Furthermore, when combined with longitudinal research design, the flexible pattern matching approach facilitates the capture of changes and processes in the investigated issues and factors (Grund and Walter 2015; Muhic and Bengtsson 2019).

To unlock the full potential of the flexible pattern matching approach, additional work is need on further refining and extending the guidelines for constructing theoretical patterns, integrating data from various sources to generate patterns and to support the matching process. Additionally, there is a need for more refined criteria or measures to assess the degree of the pattern match or mismatch and for the validation of results.

A growing set of modern, promising concepts of economic research nowadays can facilitate scholars to extract hidden relationships. For example, supported by the enormous recent progress in data storage capabilities, smart data analytics, and digital finance features (Gomber et al. 2017; McAfee and Brynjolfsson 2012) like crowdfunding, social trading, and friendship networks (Belleflamme et al. 2014; Lin et al. 2013; Pan et al. 2012) may be used to come to a better pattern matching between empirical observation and existing theories. In a similar vein, the modern concept of axiomatic risk measures (Artzner et al. 1999) provides an appealing and widely recognized theory of how application-oriented classes of (e.g., coherent, spectral, or convex) risk measures can be defined (Acerbi and Tasche 2002; Artzner et al. 1999; Föllmer and Schied 2002) and what sets of "reasonable" properties risk measures within the respective classes should fulfill. However, practical decisions resulting from the use of these axiomatic risk measures may contradict economic intuition or well-established empirical patterns. For example, risk-averse individuals, when utilizing the most prominent axiomatic risk measure of Conditional Value-at-Risk (Acerbi and Tasche 2002), may violate (Brandtner 2013) the empirical paradigm of diversification in portfolio selection (Koumou 2018; Markowitz 1952), increase risk exposure even though their risk aversion is expected to rise (Brandtner and Kürsten 2015), or expand risky investment though the threat of some additional background risk looms (Brandtner 2018). In any of these behavioral inconsistencies between theoretical and observed patterns, there is the need for ongoing data collection and 
adaption of the framework in order to see how the contextual boundaries of both theoretical risk measure concepts and observed empirical behavior in practical situations of decision making under risk could be adjusted accordingly. We hope that the extensive discussion of the method in this article will stimulate its adoption.

Funding Not applicable.

\section{Compliance with ethical standards}

Conflict of interest The authors declare that they have no conflicts of interest.

Open Access This article is licensed under a Creative Commons Attribution 4.0 International License, which permits use, sharing, adaptation, distribution and reproduction in any medium or format, as long as you give appropriate credit to the original author(s) and the source, provide a link to the Creative Commons licence, and indicate if changes were made. The images or other third party material in this article are included in the article's Creative Commons licence, unless indicated otherwise in a credit line to the material. If material is not included in the article's Creative Commons licence and your intended use is not permitted by statutory regulation or exceeds the permitted use, you will need to obtain permission directly from the copyright holder. To view a copy of this licence, visit http://creativecommons.org/licen ses/by/4.0\%.

\section{References}

Acerbi C, Tasche D (2002) On the coherence of expected shortfall. J Bank Finance 26(7):1487-1503

Ainsworth S, Cox JW (2003) Families divided: Culture and control in small family business. Organ Stud 24(9):1463-1485

Allison GT, Zelikow P (1971) Essence of decision: explaining the Cuban missile crisis. Little, Brown, Boston

Alvesson M, Kärreman D (2007) Constructing mystery: empirical matters in theory development. Acad Manag Rev 32(4):1265-1281

Artzner P, Delbaen F, Eber J-M, Heath D (1999) Coherent measures of risk. Math Finance 9(3):203-228

Bansal P, Corley K (2012) Publishing in AMJ - part 7: what's different about qualitative research? Acad Manag J 55(3):509-513

Barberis N, Thaler R (2002) A survey of behavioral finance. In Constantinides GM, Harris M, Stulz RM (eds) Handbook of the economics of finance, vol 1. Elsevier, pp 1053-1128

Baxter RJ, Berente N (2010) The process of embedding new information technology artifacts into innovative design practices. Inf Organ 20(3-4):133-155

Belleflamme P, Lambert T, Schwienbacher A (2014) Crowdfunding: tapping the right crowd. J Bus Ventur 29(5):585-609

Bhatti MW, Ahsan A (2016) Global software development: an exploratory study of challenges of globalization, HRM practices and process improvement. RMS 10:649-682

Bitektine A (2007) Prospective case study design: Qualitative method for deductive theory testing. Organ Res Methods. https://doi.org/10.1177/1094428106292900

Bitektine A (2008) Prospective case study design-qualitative method for deductive theory testing. Organ Res Methods 11(1):160-180

Black F, Scholes M (1973) The pricing of options and corporate liabilities. J Polit Econ 81(3):637-654

Bouncken R, Barwinski R (2020) Shared digital identity and rich knowledge ties in global 3d printing-a drizzle in the clouds? Glob Strategy J. https://doi.org/10.1002/gsj.1370

Bouncken RB, Laudien SM, Fredrich V, Görmar L (2018) Coopetition in coworking-spaces: value creation and appropriation tensions in an entrepreneurial space. RMS 12(2):385-410 
Bouncken R, Qiu Y, García FJS (2021) Flexible pattern matching approach: suggestions for augmenting theory evolvement. Technol Forecast Soc Change (Accepted)

Brandtner M (2013) Conditional value-at-risk, spectral risk measures and (non-)diversification in portfolio selection problems - a comparison with mean-variance analysis. J Bank Finance 37(12):5526-5537

Brandtner M (2018) Expected shortfall, spectral risk measures, and the aggravating effect of background risk, or: risk vulnerability and the problem of subadditivity. J Bank Finance 89:138-149

Brandtner M, Kürsten W (2015) Decision making with expected shortfall and spectral risk measures: the problem of comparative risk aversion. J Bank Finance 58:268-280

Brooks J, King N (2014) Doing template analysis: evaluating an end-of-life care service. SAGE Publications, Thousand Oaks

Brooks J, McCluskey S, Turley E, King N (2015) The utility of template analysis in qualitative psychology research. Qual Res Psychol 12(2):202-222

Burchell N, Kolb D (2003) Pattern matching organisational cultures. J Manag Organ 9(3):50-61

Burton J, Khammash M (2010) Why do people read reviews posted on consumer-opinion portals? J Mark Manag 26(3-4):230-255

Canato A, Ravasi D, Phillips N (2013) Coerced practice implementation in cases of low cultural fit: cultural change and practice adaptation during the implementation of six sigma at 3m. Acad Manag J 56(6):1724-1753

Cassell C, Bishop V (2019) Qualitative data analysis: exploring themes, metaphors and stories. Eur Manag Rev 16(1):195-207

Cassell C, Symon G, Buehring A, Johnson P (2006) The role and status of qualitative methods in management research: an empirical account. Manag Decis 44(2):290-303

Cassell C, Cunliffe AL, Grandy G (2018) Introduction: Qualitative research in business and management. In: Cassel C, Cunliffe A, Grandy G (eds) The sage handbook of qualitative business and management research methods. SAGE Publications, Thousand Oaks, pp 1-13

Chiles TH, Bluedorn AC, Gupta VK (2007) Beyond creative destruction and entrepreneurial discovery: a radical austrian approach to entrepreneurship. Organ Stud 28(4):467-493

Chiles TH, Meyer AD, Hench TJ (2010) Organizational emergence: the origin and transformat of branson, missouri's musical theaters. Organ Sci 19(6):907-918

Corsaro D, Snehota I (2010) Searching for relationship value in business markets: are we missing something? Ind Mark Manag 39(6):986-995

Crabtree BF, Miller WL (1999) Using codes and code manuals: a template organizing style of interpretation. In: Crabtree BF, Miller WL (eds) Doing qualitative research. SAGE Publications, Thousand Oaks, pp 163-177

Cuervo-Cazurra A, Andersson U, Brannen MY, Nielsen BB, Reuber AR (2016) From the editors: can i trust your findings? Ruling out alternative explanations in international business research. J Int Bus Stud 47:881-897

De Long JB, Shleifer A, Summers LH, Waldmann RJ (1990) Noise trader risk in financial markets. J Polit Econ 98(4):703-738

Eisenhardt KM (1989) Building theories from case study research. Acad Manag Rev 14(4):532-550

Eisenhardt KM, Graebner ME (2007) Theory building from cases: opportunities and challenges. Acad Manag J 50(1):25-32

Föllmer H, Schied A (2002) Convex measures of risk and trading constraints. Finance Stochast 6(4):429-447

Gatignon A, Capron L (2020) The firm as an architect of polycentric governance: building open institutional infrastructure in emerging markets. Strateg Manag J. https://doi.org/10.1002/smj.3124

Gioia DA (2004) A renaissance self: prompting personal and professional revitalization. In: Stablein RE, Frost PJ (eds) Renewing research practice. Stanford University Press, Stanford, pp 97-114

Gioia DA, Price KN, Hamilton AL, Thomas JB (2010) Forging an identity: an insider-outsider study of processes involved in the formation of organizational identity. Adm Sci Q 55(1):1-46

Gioia DA, Corley KG, Hamilton AL (2013) Seeking qualitative rigor in inductive research: notes on the gioia methodology. Organ Res Methods 16(1):15-31

Gittins T, Lang R, Sass M (2015) The effect of return migration driven social capital on SME internationalisation: a comparative case study of it sector entrepreneurs in Central and Eastern Europe. RMS 9:385-409

Glaser BG, Strauss AL (1968) The discovery of grounded theory: strategies for qualitative research. Nurs Res 17(4):364-366

Gomber P, Koch J-A, Siering M (2017) Digital finance and fintech: current research and future research directions. J Bus Econ 87(5):537-580 
Graebner ME, Martin JA, Roundy PT (2012) Qualitative data: cooking without a recipe. Strateg Organ 10(3):276-284

Grbich C (2013) Qualitative data analysis: an introduction. SAGE Publications, Los Angeles

Greenwood R, Hinings CR, Brown J (1994) Merging professional service firms. Organ Sci 5(2):239-257

Grund C, Walter T (2015) Management compensation and the economic crisis: longitudinal evidence from the German chemical sector. RMS 9:751-777

Hammond KR (1966) Probabilistic functionalism: Egon brunswick's integration of the history, theory, and method of psychology. In: Hammond KR (ed) The psychology of Egon Brunswik. Holt, Rinehart and Winston, Inc, New York, pp 15-80

Harrison JM, Kreps DM (1979) Martingales and arbitrage in multiperiod securities markets. J Econ Theory 20(3):381-408

Hiebl MRW, Mayrleitner B (2019) Professionalization of management accounting in family firms: the impact of family members. RMS 13:1037-1068

Idemen E, Elmadag AB, Okan M (2020) A qualitative approach to designer as a product cue: proposed conceptual model of consumers perceptions and attitudes. Rev Manag Sci. https://doi.org/10.1007/ s11846-020-00381-5

Jancsary D, Meyer RE, Höllerer MA, Barberio V (2017) Toward a structural model of organizationallevel institutional pluralism and logic interconnectedness. Organ Sci 28(6):1150-1167

Jobber D, Lucas GJ (2000) The modified tichy tpc framework for pattern matching and hypothesis development in historical case study research. Strateg Manag J 21(8):865-874

Kauppila OP (2010) Creating ambidexterity by integrating and balancing structurally separate interorganizational partnerships. Strateg Organ 8(4):283-312

Kenealy GJJ (2012) Grounded theory: a theory building approach. In: Symon G, Cassell C (eds) Qualitative organizational research core methods and current challenges. SAGE, London, pp 408-425

King N (2004) Using templates in the thematic analysis of text. In: Cassell C, Symon G (eds) Essential guide to qualitative methods in organizational research, vol 256. SAGE Publications, London

King N, Brooks J, Tabari S (2018) Template analysis in business and management research. In: Ciesielska M, Jemielniak D (eds) Qualitative methodologies in organization studies, vol 2. Palgrave Macmillan, Cham, pp 179-206

Koumou GB (2018) Diversification and portfolio theory: a review. Rev Req Financ Mark Portf Manag 34:267-312

Langley A (1999) Strategies for theorizing from process data. Acad Manag Rev 24(4):691-710

Langley A, Abdallah C (2011) Templates and turns in qualitative studies of strategy and management. In: Bergh DD, Ketchen DJ (eds) Building methodological bridges. Emerald Group Publishing Limited, pp 201-235

Lee AS (1989) A scientific methodology for MIS case studies. MIS Q Manag Inf Syst 13(1):33-50

Lin M, Prabhala NR, Viswanathan S (2013) Judging borrowers by the company they keep: friendship networks and information asymmetry in online peer-to-peer lending. Manag Sci 59(1):17-35

Markowitz H (1952) Portfolio selection. J Finance 7:77-91

Massaro M, Moro A, Aschauer E, Fink M (2017) Trust, control and knowledge transfer in small business networks. RMS 13(2):267-301

McAfee A, Brynjolfsson E (2012) Big data: the management revolution. Harv Bus Rev 90(10):51-68

McCutcheon DM, Meredith JR (1993) Conducting case study research in operations management. J Oper Manag 11(3):239-256

Montealegre R (2002) A process model of capability development: Lessons from the electronic commerce strategy at bolsa de valores de guayaquil. Organ Sci 13(5):514-531

Muhic M, Bengtsson L (2019) Dynamic capabilities triggered by cloud sourcing: a stage-based model of business model innovation business model innovation. Rev Manag Sci. https://doi.org/10.5465/ AMBPP.2019.15991abstract

Ostrom E (2005) Understanding institutional diversity. Princeton University Press, Princeton

Pan W, Altshuler Y, Pentland A (2012) Decoding social influence and the wisdom of the crowd in financial trading network. Paper presented at the International Conference on Privacy, Security, Risk and Trust, Amsterdam

Parker M (1995) Working together, working apart: Management culture in a manufacturing firms. Sociol $\operatorname{Rev}$ 3:519-547

Pratt MG (2009) From the editors: For the lack of a boilerplate: Tips on writing up (and reviewing) qualitative research. Acad Manag J 52(5):856-862

Reay T, Jones C (2016) Qualitatively capturing institutional logics. Strateg Organ 14(4):441-454 
Rindova VP, Petkova AP, Kotha S (2007) Standing out: How new firms in emerging markets build reputation. Strateg Organ 5(1):31-70

Ross SA (1978) A simple approach to the valuation of risky streams. J Bus 51(3):453-475

Ross J, Staw BM (1993) Organizational escalation and exit: Lessons from the shoreham nuclear power plant. Acad Manag J 36(4):701-732

Saka A (2004) The cross-national diffusion of work systems: Translation of Japanese operations in the UK. Organ Stud 25(2):209-228

Sang KJC, Sitko R (2015) Qualitative data analysis approaches. In: O’Gorman K, MacIntosh R (eds) The, 2nd edn. Goodfellow Publishers, London, pp 140-154

Shane S (2000) Prior knowledge and the discovery of entrepreneurial opportunities. Organ Sci 11(4):448-469

Shleifer A, Vishny RW (1997) The limits of arbitrage. J Finance 52(1):35-55

Sinkovics N (2016) Enhancing the foundations for theorising through bibliometric mapping. Int Mark Rev 33(3):327-350

Sinkovics N (2018) Pattern matching in qualitative analysis. In: Cassel C, Cunliffe A, Grandy G (eds) The sage handbook of qualitative business and management research methods. SAGE Publications, Thousand Oaks, pp 468-485

Sinkovics N, Sinkovics RR, Yamin M (2014) The role of social value creation in business model formulation at the bottom of the pyramid-implications for MNEs? Int Bus Rev 23(4):692-707

Sinkovics N, Hoque SF, Sinkovics RR (2016) Rana Plaza collapse aftermath: are CSR compliance and auditing pressures effective? Acc Audit Account J 29(4):617-649

Sinkovics N, Choksy US, Sinkovics RR, Mudambi R (2019) Knowledge connectivity in an adverse context: Global value chains and Pakistani offshore service providers. Manag Int Rev 59(1):131-170

Soto-Simeone A, Kautonen T (2020) Senior entrepreneurship following unemployment: a social identity theory perspective. Rev Manag Sci. https://doi.org/10.1007/s11846-020-00395-z

Spurrell M, Araujo L, Proudlove N (2019) Capturing context: An exploration of service delivery networks in complex case management. Ind Mark Manag 76:1-11

Strauss A, Corbin J (1998) Basics of qualitative research: Techniques and procedures for developing grounded theory, 2nd edn. SAGE Publications, Thousand Oaks

Thornton PH, Ocasio W, Lounsbury M (2012) The institutional logics perspective: a new approach to culture, structure, and process. Oxford University Press, Oxford, USA

Tichy NM (1983) Managing strategic change: technical, political, and cultural dynamics. Wiley, New York.

Trochim WMK (1985) Pattern-matching, validity, and conceptualization in program-evaluation. Eval Rev 9(5):575-604

Trochim WMK (1989) Outcome pattern matching and program theory. Eval Program Plan 12(4):355-366

Trochim WMK, Donnelly JP (2008) Research methods knowledge base. Atomic Dog/Cengage Learning, Mason

Van de Ven AH, Huber GP (1990) Longitudinal field research methods for studying processes of organizational change. Organ Sci 1(3):213-219

Waring T, Wainwright D (2008) Issues and challenges in the use of template analysis: two comparative case studies from the field. Electron J Bus Res Methods 6(1):85-94

Yin RK (1994b) Discovering the future of the case study. Method in evaluation research. Eval Pract 15(3):283-290

Yin RK (1994a) Case study research and applications: Design and methods. SAGE Publications, Thousand Oaks

Yin RK (2009) Case study research: design and methods. SAGE Publications, Los Angeles

Yin RK, Moore GB (1988) Lessons on the utilization of research from nine case experiences in the natural hazards field. Knowl Soc 1(3):25-44

Zalewska-Kurek K, Harms R (2020) Managing autonomy in university-industry research: a case of collaborative ph.D. Projects in the Netherlands. RMS 14:393-416

Zardini A, Ricciardi F, Bullini Orlandi L, Rossignoli C (2020) Business networks as breeding grounds for entrepreneurial options: organizational implications. RMS 14(5):1029-1046

Publisher's Note Springer Nature remains neutral with regard to jurisdictional claims in published maps and institutional affiliations. 